tron beam gives a rate of $\sim 2 \times 10^{8}$ positrons per second.

The investigators see possible applications in positron-annihilation and Doppler-broadening spectroscopy, as well as positronium spectroscopy.

TIM PALUCKA

\section{Scanning Calorimetry Technique Extended to Nanoliter-Scale Samples}

Conventional differential scanning calorimetry (DSC), a technique for measuring heat exchange during chemical reactions or phase transformations, is limited to milliliter-sized, macroscopic samples. However, a team of researchers in the Materials Department at the University of Illinois at UrbanaChampaign fabricated a calorimeter for use with solid and liquid samples possessing volumes of the order of a few nanoliters. The calorimeter consists of a $0.3-\mu \mathrm{m}$-thick silicon nitride membrane on a silicon frame. A small "box" (volume $\sim 35 \mathrm{~nL}$ ) is on one side of the membrane, and a nickel heating line is on the other.
In the October 23 issue of Applied Physics Letters, L.H. Allen, E.A. Olson, S. Lai, J.T. Warren, and co-workers report the test outcome of their "biobox" nanocalorimeter, which was fabricated at the Cornell Nanofabrication Facility. For both indium and water-droplet specimens, they demonstrated excellent agreement between both measured melting point and heat of vaporization values and the accepted values.

Calorimeter performance was evaluated in scanning and heat-conductive modes for liquid and solid samples. In the scanning mode (100-150 K/s), the melting point of a $52 \mathrm{~nL}$ indium specimen and the heat of vaporization of water droplets (2-100 mL) were determined by heatcapacity measurements. The results demonstrate that, in this mode, the system operates with a temperature sensitivity of $\pm 0.1 \mathrm{~K}$ and power sensitivity of $\pm 7 \mu \mathrm{W}$. In the heat-conductive mode, which is especially useful for processes that occur near room temperature, a $\sim 60 \mathrm{~nL}$ water droplet was placed in the calorimeter, and the system temperature was monitored as the droplet evaporated. The experimental heat of vaporization fell within $25 \%$ of the predicted value, and the system's temperature and power sensitivity were shown to be $\pm 13 \mathrm{mK}$ and $\pm 3 \mu \mathrm{W}$, respectively.

According to Allen, with the extension of calorimetry to nanoliter-scale materials, scientists may be able to gain insight into the thermodynamics and kinetics of biological processes and nanoscale materials, areas in which recent interest has soared. Fields in which nanocalorimetry may find application include the biological sciences in studies of basic cell processes and the microelectronics industry in studies involving individual flipchip solder balls.

"Amazing things happen when the size of the matter is on the nanometer scalefor example, the melting point dramatically decreases," said Allen. Using the new nanocalorimetry device, Zhang, Kwan, Wisleder, and co-workers report in the October 15 issue of Physical Review B that when the size of indium particles is of the order of a few thousand atoms, they melt at room temperature. "Even more amaz-

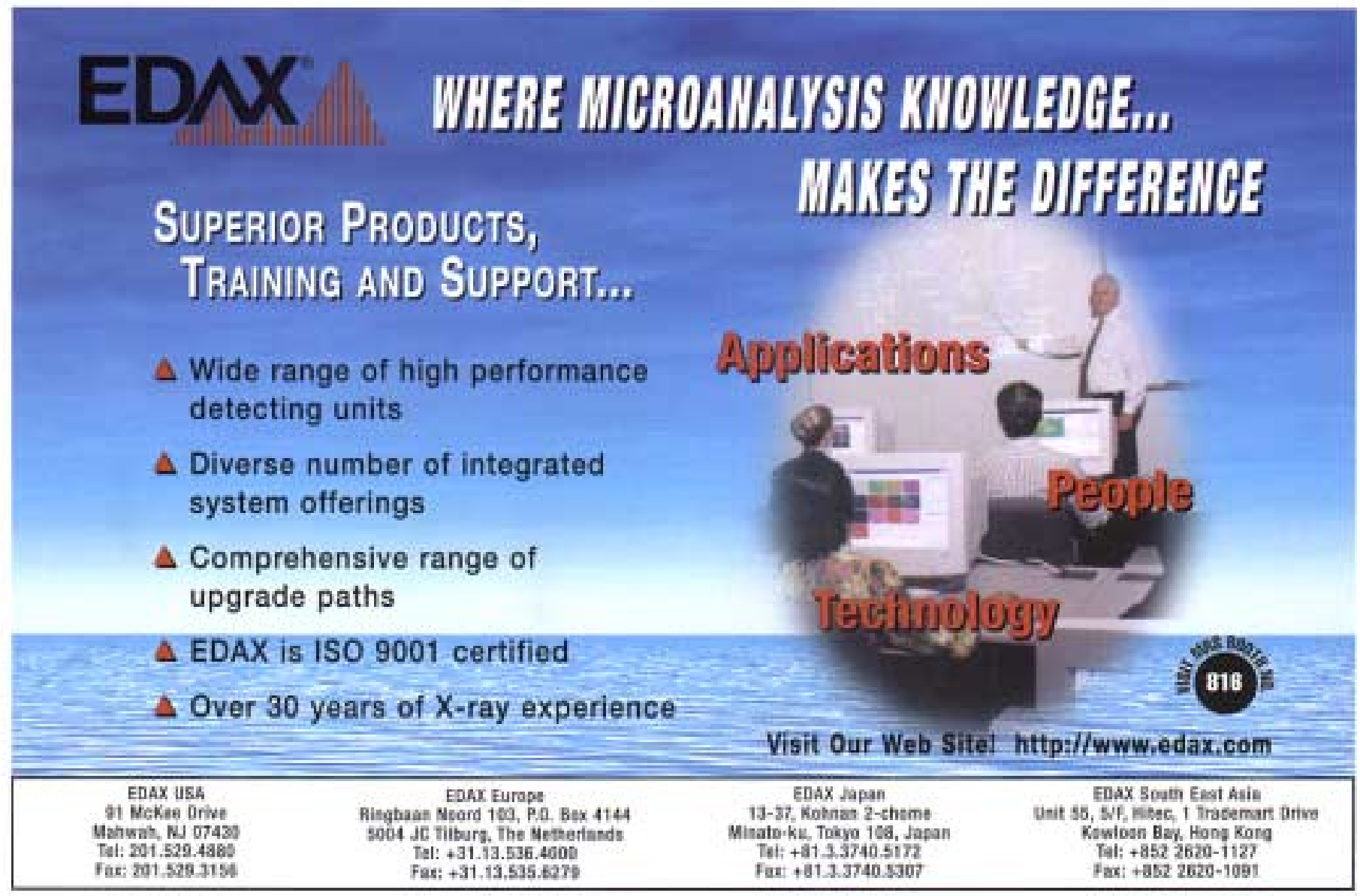

Circle No, 6 on Inside Back Cover 
ing is that nanoscale indium particles seem to self-organize into specific sizesmagic number sizes," Allen said. In the October 23 issue of Physical Review Letters, Efremov, Schiettekatte, and co-workers show, using nanocalorimetry, that the particles appear to self-assemble into special "magic" sizes, where each size differs incrementally by a single complete shell of atoms.

STEFFEN K. KALDOR

\section{Diffraction-Free Propagation of Rayleigh Waves Observed along Solid Spherical Surfaces}

Vibration and surface acoustic wave propagation in elastic spheres has been studied extensively, especially in seismology, where an elastic sphere is viewed as the simplest model of the Earth. Recent experiments have shown that the method by which surface waves propagate along a sphere depends on whether they arise from a point source or a line source. In seismology studies, where the source is orders of magnitude smaller than the Earth's radius, a point source assumption is valid, and the wave behavior is well understood. However, researchers Yusuke Tsukahara and Noritaka Nakaso of Toppan Printing Company Ltd. and Hideo Cho and Kazushi Yamanaka of the Department of Materials Processing at Tohoku University observed earlier this year that as the radius of the sphere becomes comparable in size to the source, markedly different wave-propagation behavior occurs that cannot be explained by a point-source analysis, suggesting diffraction-free propagation [Appl. Phys. Lett., 76 (2000) p. 2797]. Now, in the October 30 issue of Applied Physics Letters, the researchers present a model, along with supporting experimental evidence, to explain this observed phenomenon.

When a point force is applied to the surface of an elastic sphere at the south pole, Rayleigh waves, in addition to bulk waves, propagate along the surface in all directions. As the wave front crosses the equator, it converges toward the north pole and then diverges back toward the south pole; this process repeats indefinitely. Experiments carried out with a 20-mm-diameter sphere heated by a laser beam focused to a small spot demonstrate this behavior. However, experiments employing an 8$\mathrm{mm}$-diameter sphere heated by a line source consisting of a traveling optical inteference pattern show surface waves which travel within a narrow path over the spherical surface rather than spreading out, that is, diffraction-free propagation.

To explain this phenomenon, Tsukahara and co-workers postulate that a line source of finite length, located along the equator, generates a beam of Rayleigh waves that undergo two effects that balance, if the line source length is appropriately chosen. The first effect, due to the curvature of the spherical surface, causes the Rayleigh waves to converge toward the poles, while the second effect, diffraction from a finite aperture, causes the Rayleigh waves to spread out over the surface of the sphere. When these two effects cancel, diffractionfree propagation of a finite beam width is realized.

To verify their theory, the researchers fabricated and polished an 80-mm-diameter glass ball. An ultrasonic transducer

ADVANGED MIGROANALYYSIS TEGHNOLOGY AJ AN AFFORDABLE PRIGE

MIJAOAMALYZEA OFFEABS
$\triangle$ Fast Report Generation
A Same Algorithms as Phoenix
A Fully Upgradable to Phoenix
$\Delta$ Interface to Existing or New Detectors

EDAX FEATUAES:

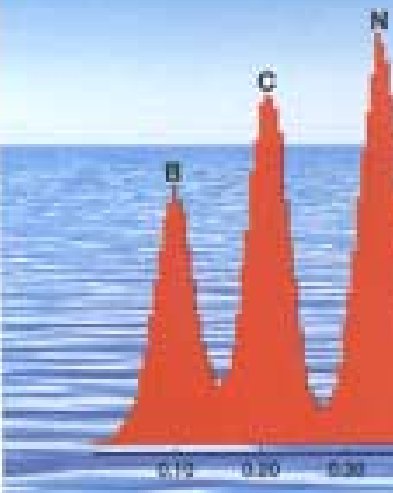

$\checkmark \mathrm{PCl}$ Bus (32 bit)

Architecture

$\checkmark$ Windows 98 or Windows NT

$\checkmark$ Digital Processing Standard

7 Superior Service \& Support
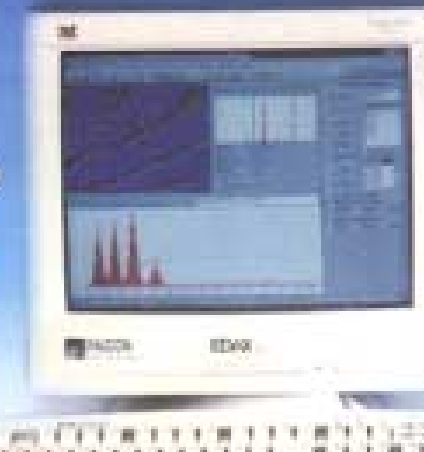

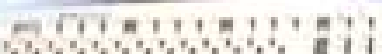



Falcon system

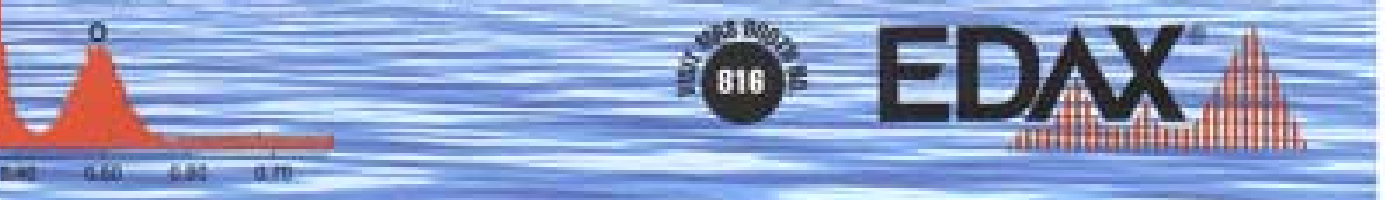

Circle No. 6 on Inside Back Cover 